nostro Ospedale è in uso il test EIA BEIA Mab Syphilis screen "back titration" (Bouty) in micropiastra che viene confermato nei casi positivi con il test TPHA (Delta) e RPR (BD). Abbiamo confrontato $\mathrm{i}$ test in uso con il Liaison Treponema Screen (DiaSorin).

Metodi: sono stati valutati 1217 sieri così suddivisi: 699 sieri della routine (pazienti interni ed esterni); 449 sieri di donatori della banca sangue, 69 sieri positivi o problematici raccolti negli anni precedenti e conservati in congelatore a $20^{\circ} \mathrm{C}$. I sieri sono stati valutati in parallelo con EIA BEIA, TPHA (routine) o TPPA (donatori), RPR e Liaison Treponema Screen (LTS). Vengono considerati discrepanti i casi che differiscono per almeno un test (discrepanza dubbia per valori entro $\pm 10 \%$ del cut-off). Nel caso di discrepanza tra EIA BEIA e LTS fa testo il TPHA (o TPPA). In 66 casi è stata eseguita la Western Blot.

Risultati: Donatori ${ }^{\circ}$ 449; Negativi su tutti i test 436; EIA BEIA da ricontrollare 12 (5 positivi, 7 dubbi); LTS da ricontrollare 1 (dubbio); TPPA tutti negativi. Routine $\mathrm{n}^{\circ}$ 699; Negativi su tutti i test 609; Positivi per tutti i test 13; da ricontrollare EIA BEIA 72 (45 positivi, 27 dubbi) ed LTS 4 (2 positivi, 2 dubbi), e tutti sono risultati TPHA negativi; Positivi solo per RPR 1. Positivi o problematici $\mathrm{n}^{\circ} 69$; concordanti EIA BEIA, LTS e TPHA 67, positivi RPR 2 (TPHA negativo dopo assorbimento, WB negativa EIA BEIA e LTS entrambi 1 positivo e 1 negativo).

Conclusioni: Nel nostro campione entrambi i test di screening hanno una elevatissima sensibilità (assenza di falsi negativi). Calcolando donatori e routine ( $\mathrm{n}^{\circ} 1148$ test) i casi da ricontrollare sono stati 5 per LTS e 84 per EIA BEIA. Nella gestione di un test che è a basso costo e ad elevata frequenza di richiesta, una maggior specificità e una più facile automazione semplificano l'organizzazione del lavoro.

\title{
VALUTAZIONE DEL TEST LIAISON TREPONEMA SCREEN
}

\author{
Rusconi N., Marincola G., Barletta R., Pichierri L.,
} Scandurra V., Vaiani R.

Microbiologia, Ospedale "A. Manzoni", A.O. Ospedale di Lecco, via dell'eremo 9/1 I, 23900 Lecco

Introduzione: lo screening per la lue è tuttora un esame molto richiesto ed è utile semplificarne la gestione. Nel 